\title{
Evaluation of Wireless Fidelity Performance in Nigeria's Mobile Networks
}

\author{
Arinze S.N. ${ }^{1}$, Onoh G. N. ${ }^{2}$ and Abonyi D.O. ${ }^{3}$ \\ $\mathbf{1 , 2}^{\mathbf{1}}$ Electrical and Electronic Engineering, Enugu State University of Science and \\ Technology, Enugu State, Nigeria \\ Corresponding author: Arinze S. N.
}

\begin{abstract}
This work focused on the Wireless Fidelity performance evaluation in Nigeria using mobile networks. The existing Wireless Fidelity System (Wi-Fi) from three different networks (MTN, 9MOBILE, and GLO) were characterized to determine the throughput, latency, and packet loss. Enugu State University of Science and Technology Exxon Mobil Laboratory was used as a case study. Ten laptop devices connected to the networks were installed with NetLimiter Software to capture the traffic statistics. The same data size of 29.12MB was downloaded from the YouTube website for three months to obtain the average network performance parameters as a result of the daily variations of the received signal strength. The result showed that there was a high decrease in data throughput, an increase in latency, and packet loss as the connected user's increases. Although out of three different networks used, MTN got the best network performance. Wi-Fi network utilizes the already congested radio waves. Other frequencies such as Visible Light Spectrum can complement with it to yield high data speed communication.
\end{abstract}

Keywords: Wireless Fidelity ( Wi-Fi), Throughput, Latency, Packet loss, Netlimiter Software, Visible Light Spectrum

\section{Introduction}

The formation of the Global System for Mobile (GSM) network into the Nigerian market was cosmically accepted and found to be efficient at the inception. With time, operators in the industry experienced remarkable growth in the customer base, which later incapacitated the networks to function efficiently. Nigerian Communications Commission (NCC) as a regulatory body of the GSM providers such as MTN, Globacom, 9Mobile, Airtel, etc in Nigeria has investigated the inefficiency of data throughput and recommended that ninety percentage of network data throughput should be experienced by the customers for a good Quality of Service (QoS) and yet the situation has not been improved [1]. The problem of data throughput is experienced all over the world, and it has become a subject for the researchers to implement a technique that will overcome the rapidly growing wireless data traffic in gadgets usage and application developments.

Authors[2] proposed a High throughput Wi-Fi Backscatter using Back-Fi technology. BackFi technology is a full-duplex radio technology that reuses standard Wi-Fi transmissions as an excitation signal to avoid co-existence issue. It then overlaps backscatter data on down-link Wi-Fi packets from the access point and improves the spectrum efficiently. Finally, it integrates the backscatter reader and $\mathrm{Wi}-\mathrm{Fi}$ access point naturally in a single device. It was found to enhance the Wi-Fi backscatter link throughput, achieving communication rates up to 
$15 \mathrm{Mbps}$ at a range of 5meters. Its throughput is low and can improve with the use of multiple antennas at the $\mathrm{Wi}-\mathrm{Fi}$ access points.

Throughput Improvement of a Range Aware Wi-Fi Network, Minimizing Signal Interference, was made by controlling the Acess Points transmission power, considering the locations of wireless devices [3]. Simulation results showed that Range Aware Wi-Fi network gave higher network throughput (of about 5\%) than conventional Wi-Fi network. Since today's Wi-Fi environment has a very high density of wireless client due to the rapid increase of Wi-Fi devices, it cannot provide a large throughput to users.

Several authors[4] presented an Adaptive Device to Device Communication using Wi-Fi Direct in smart Cities. In their work, the adaptive single presence period algorithm running on the Wi-Fi Direct estimates the time the channel is busy and then changes the presence window based on the estimated traffic utilization in the channel. It worked well but had less throughput when there was heavy traffic. This work will evaluate the performance of Wireless Fidelity in Nigeria using mobile networks.

\section{Theory}

Wireless Fidelity (Wi-Fi) is a technology that utilizes Radiofrequency for exchange of information in Local Area Network (LAN) without cable and wiring. It was brought under the name WAVELAN in the 1980s and further developed into IEEE 802.11 standard in 1997 [5]. The standard supported only $2 \mathrm{Mbps}$ connections which were too slow until new versions of standard such as $802.11 \mathrm{a}, 802.11 \mathrm{~b}, 802.11 \mathrm{~g}, 802.11 \mathrm{n}$, and so on were developed [6]. Wi-Fi is an open standard technology, and its operation is in the unlicensed $2.4 \mathrm{GHz}$ radio band with $11 \mathrm{mbps}$ or $54 \mathrm{mbps}$ data rate and ten times faster than the regular dial-up connection [6]. WiFi technology was widely accepted and integrated into digital products to realize a wireless digital network. Bahl first used it in 2000 for indoor positioning. Later on, in 2009, it was introduced in subway systems and used as an outdoor location technology by Nobuo Kawaguchi [7]. Wireless Fidelity has a broad availability of terminal devices in the cordless phone, camera, media players, personal data assistants, and laptops. Its connectivity to the internet can be from anywhere in the world, like a hotel room, conference room, coffee shop, pharmaceutical shops, etc. Networking of Wi-Fi is possible through three elements, which are Radio signals, Access point, and Network interface card.

- Radio Signals are electromagnetic waves of $30-300 \mathrm{GHz}$ frequencies used to establish Wi-Fi connections for the transmission of messages. They are generated artificially by transmitters and received by radio receivers such as an antenna. It travels at the speed of light.

- Access Points (AP) are the Physical / Data link layer devices that act as a base station, inform the wireless client, authenticate and associate them with the network or behave as an operating hub to maintain wireless connectivity between the computers. They are the primary source that transmits and receive waves as well as access network services such as email, web browsing, and database application. They consist of antennas and routers. Antennas are mainly used for longer radio transmission while routers are suitable for homes. AP supports the most usable 1, 2, 5.5, or $11 \mathrm{Mbps}$ connectivity.

- Network Interface Card (NIC) is a wireless controller used by Personal Computers (PC) to scan the available spectrum and associates it to the access point for connectivity. Generally, it fits inside the computer device and provides the interface between the PC and wireless network. NIC has to match wireless network infrastructure for communication access with each other. Examples of NIC are Peripheral Component Interconnect (PCI) card, Personal Computer (PC) card, and 
Compact Flash (CF) card. They get packets ready for sending, receiving, and convert data transmission from parallel to serial.

The radio signals transmitted from antenna and routers are picked up by NIC cards in computers and cell phones, then create wireless internet connection between the user and the network. However, the reception of the signal has to fall within the range of 46-92 meters for antennas, which are a general rule of thumb in Wi-Fi home networking. The speed of the WiFi connection increases as it gets closer to the router but decreases as it gets farther away. Its operational range depends on radio power output, receiver sensitivity, frequency band, antenna type, antenna gain, and modulation technique. The connection can be through the access point to client or client to client as shown in figure 1

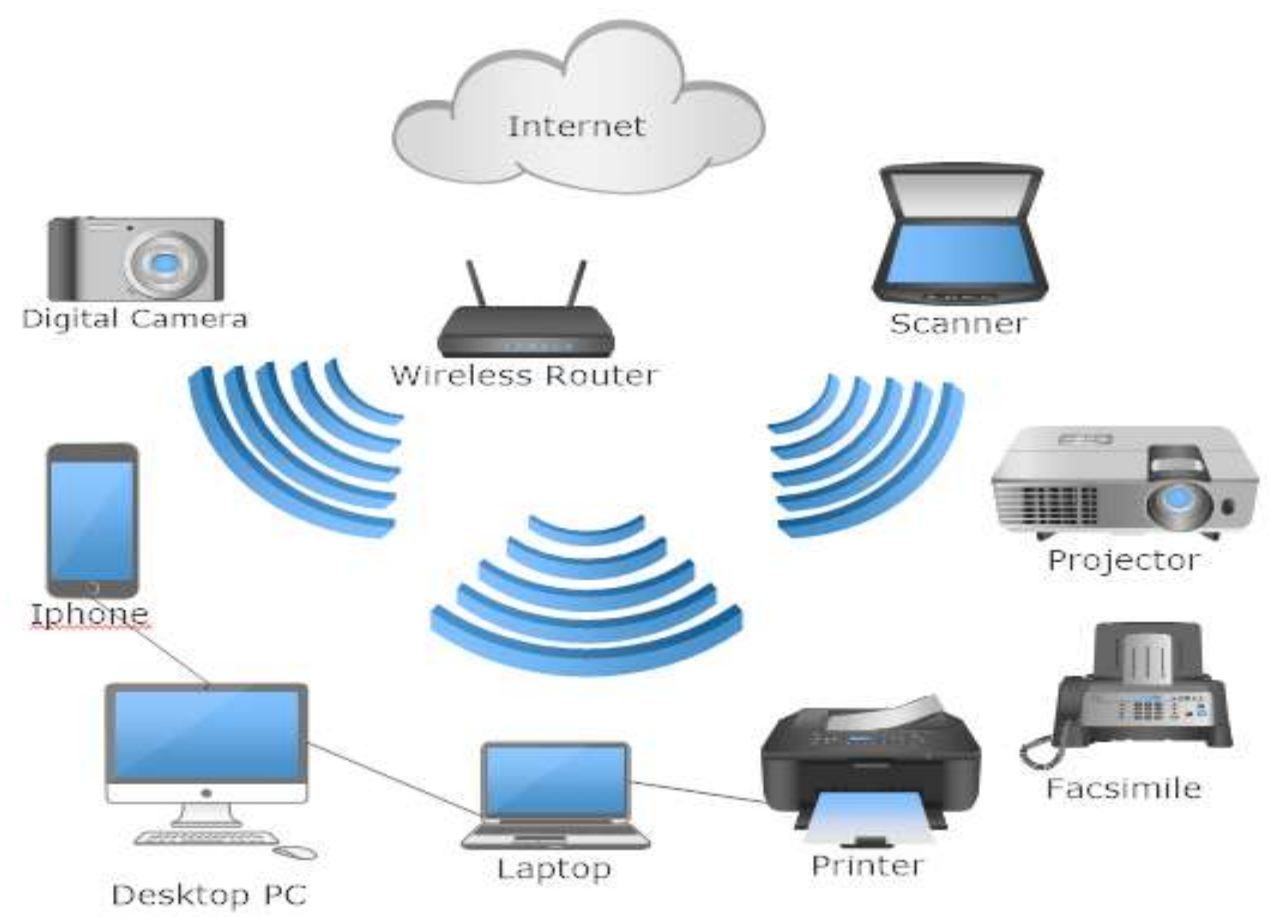

Figure 1: Wi-Fi Connection (ConceptDraw, 2015)

\section{Network Performance}

Network Performance is the reviewing of network metrics to define the quality of services offered by the computer network. It is measured from the end-user and is by analyzing the statistics from the following network parameters

- Throughput: This is the amount of data successfully sent or received in a given time. Its measured in bit per second (bps), byte per second (Bps) or data packets per second(pps). Throughput can be used to measure the performance of internet and network connections through transferring of a large file, obtaining the time required for the download of the file and divide the file size by the time. Factors affecting throughput are multi-user connections, hardware, bandwidth, Signal to noise ratio (SNR), and bit error rate (BER). Therefore, effective design in considering these factors will drive its final performance.

- Latency: This is the time required to transmit a packet across a network. It can measure as one way (the time the source sends a data packet to the receiver) or round trip delay time (addition of one way latency from source to destination and a destination to source). A high latency degrades the performance of the network while a low latency drives the maximum throughput of a conversation. 
- Packet Loss: These are packets lost in transmission and delivery. Due to network congestion, one or more packet of data might fail to reach its destination. The dropped packets can retransmit at the expense of a decrease in throughput.

- BER: This is the percentage of bits that have errors relative to the total number of bits sent. Factors affecting it are interference, modulation scheme, increased bandwidth, and noise.

- SNR: This is the ratio between signal power and noise power. Its represented in decibels $(\mathrm{dB})$

- Bandwidth: This is the maximal amount of data that can travel through a channel or the available data transfer

\section{Materials and Method}

Characterization of the existing Wi-Fi system was done to determine the network performance when many devices connect to it. This goal can be achieved using the extended ping method, which gives the user the option of specifying the internet source address of the data packet. Unfortunately, in using this method, one needs to lower the packet size and hence, measuring network throughput through this means is unreliable. Utility tools such as NetLimiter software which is a full internet traffic control and monitory tool over the computer was used instead of the extended ping method because it can measure any data size with a high degree of accuracy [8]

The experimental setup, as shown in figure 2, was used in the Wi-Fi network characterization.

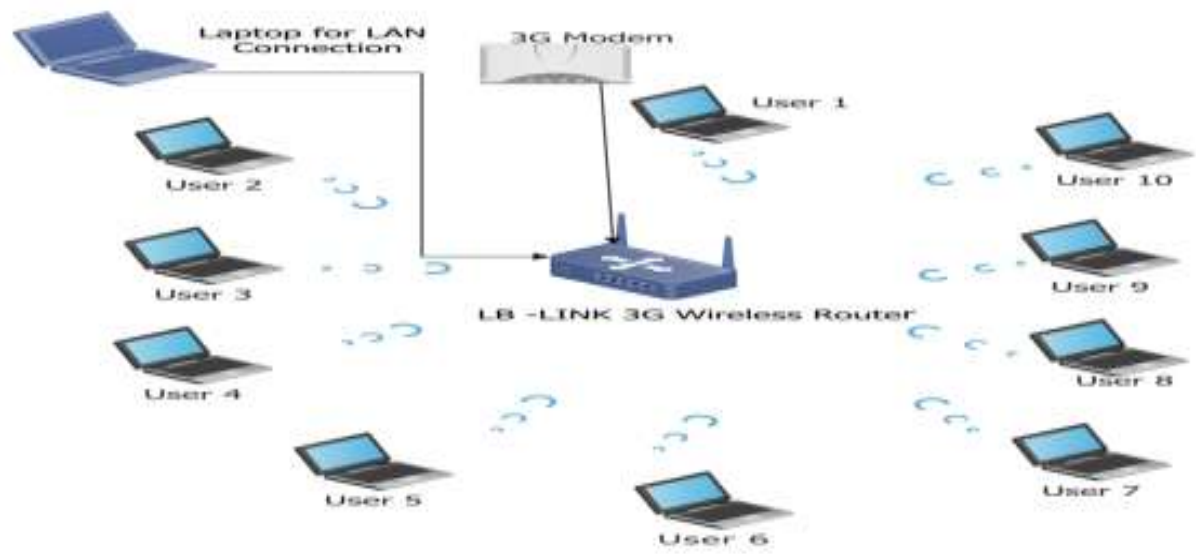

Figure 2: Experimental Setup for determining the data throughput of Wi-Fi system

The experiment was carried out in ESUT EXXON MOBIL Laboratory. It was done for three months (June, July, August 2018) from Monday to Friday, to obtain the average network performance parameters as a result of the daily variations of the received signal strength. Materials used for the experiment were Ten laptops, and a Fourth Generation (4G) Modem of three different wireless networks (MTN, 9MOBILE, and GLO). The modem connects to the LB-LINK Wi-Fi router through the USB port. The LB-LINK Wi-Fi router LAN port was connected to the LAPTOP using an RJ45 network cable. It was configured by connecting to its portal on the Mozilla web browser using its IP address. The $4 \mathrm{G}$ parameter was set up as a means of access to the router. The access point name, username, and password of the three different wireless networks were used to gain a connection to the internet. The security key was enabled to have control of the connected users.

$4 \mathrm{G}$ Operation mode set as a gateway means the first Ethernet port is WAN port. The other Ethernet ports and the wireless interface were bridged together and treated as LAN ports for other devices access to the Wi-Fi router. The wireless network name (Service Set Identifier 
(SSID)) makes it easier for users to connect when scanning the available networks. Frequency channel was auto-selected while network mode was chosen as $11 \mathrm{~b} / \mathrm{g} / \mathrm{n}$ mixed mode to keep compatibility. The router restarted so that it will take the effect of the configurations done. All the users (ten laptops) have Wi-Fi connectivity. The NetLimiter software was installed in each of the laptops to capture the traffic statistics when downloading data over the internet. User1 was connected to the Wi-Fi router network name by logging in using the password key. It connected to the internet through Mozilla Firefox. Throughput of $29.12 \mathrm{MB}$ data downloaded once per day from the YouTube website was measured. The measurement process flow explained is shown in figure 3

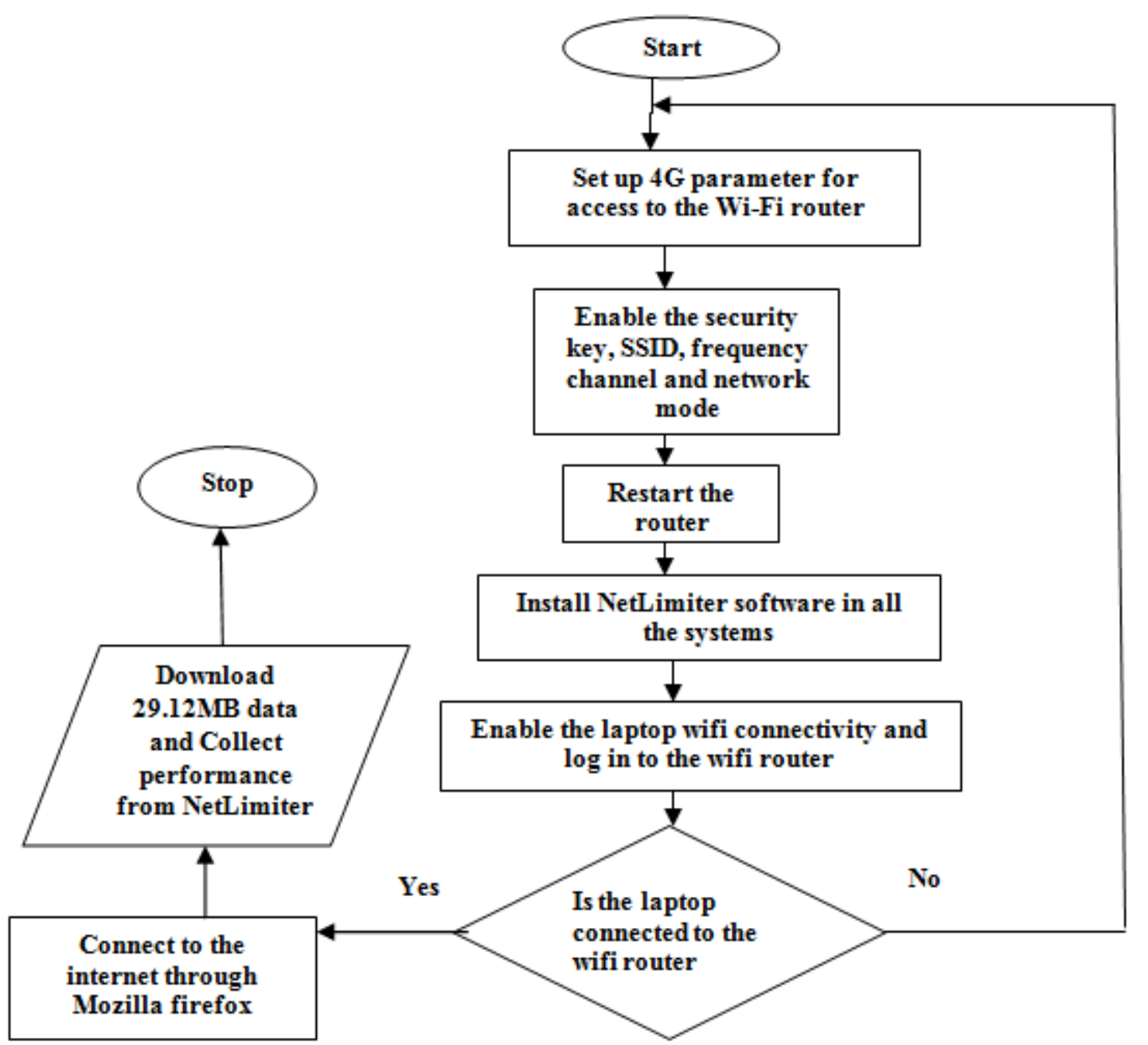

Figure 3: Flow Process showing step by step approach to setting up the testbed for throughput measurement

User1 and User2 downloaded the same data at the same time. User2 traffic statistics from NetLimiter software was captured and saved. The traffic statistics obtained for User3 was carried out with both User1 and User2 downloading the same data. The process continued until it gets to User10. Evaluation of latency, packet loss, and the bit error rate took the next step.

Data throughput was calculated using the following equation

$$
\text { Data throughput }=\frac{\text { Actual data transferred }}{\text { Time }}
$$


Graphical analysis of Latency, Packet loss, Data throughput was carried out using Python 3.6.1 64Bit. The flow chart for latency is the same for packet loss and represented below

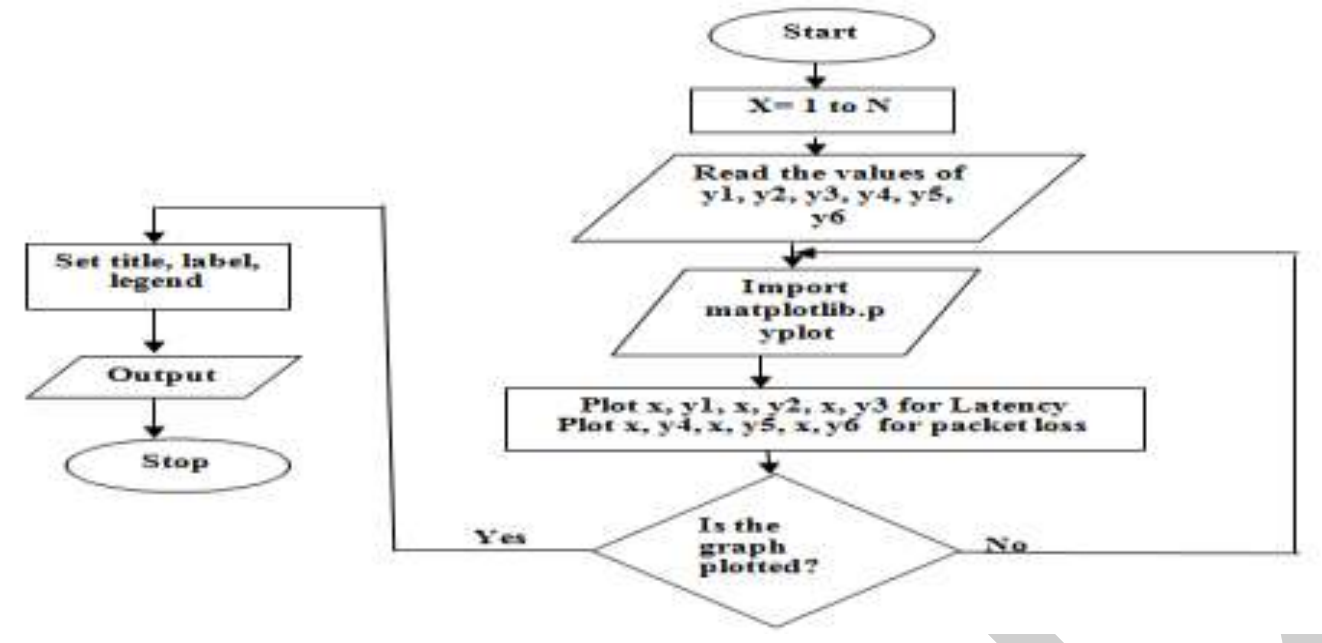

Figure 4: Plotting process of latency and packet loss

Figure 4 sets the number of laptop users (x) and reads in the values of latency $(\mathrm{y} 1, \mathrm{y} 2, \mathrm{y} 3)$ or packet loss $(\mathrm{y} 4, \mathrm{y} 5, \mathrm{y} 6)$ of the three different networks. Matplot library imported from the python environment helped to plot the values of latency, packet loss against the laptop users. The flowchart for throughput follows the same process of figure 4 except that the formula in equation (1) for calculating the throughput of the three networks was keyed in after the computer reads the values of the actual data transferred $(\mathrm{x} 1, \mathrm{x} 2, \mathrm{x} 3)$ and time $(\mathrm{y} .1, \mathrm{y} .2, \mathrm{y} .3)$ for the three different networks.

\section{Results}

The three months average network performance parameters of the Wi-Fi network which are the Latency, Packet loss and Throughput outputs obtained from Netlimiter software for the data transfer size of $29.12 \mathrm{MB}$ of the three mobile networks are shown in tables $1,2,3$ respectively

Table 1: Average Monthly Latency in seconds for data transfer of 29.12MB using three Mobile Networks

\begin{tabular}{llll}
\hline & 4G NETWORK & & \\
\hline Users & MTN & 9MOBILE & GLO \\
\hline User1 & 0.5359 & 0.7001 & 0.8053 \\
User2 & 1.5071 & 2.2952 & 2.7471 \\
User3 & 3.4512 & 5.488 & 6.6294 \\
User4 & 6.363 & 10.2764 & 12.4548 \\
User5 & 10.2475 & 16.664 & 20.2180 \\
User6 & 15.0991 & 24.653 & 29.9236 \\
User7 & 20.9238 & 34.2893 & 41.5613 \\
User8 & 27.7157 & 45.4416 & 55.1467 \\
User9 & 35.4783 & 58.1664 & 70.6671 \\
User10 & 44.2102 & 72.5344 & 88.1256 \\
\hline
\end{tabular}


Figure 5 showed the latency of the three mobile networks obtained in table 1

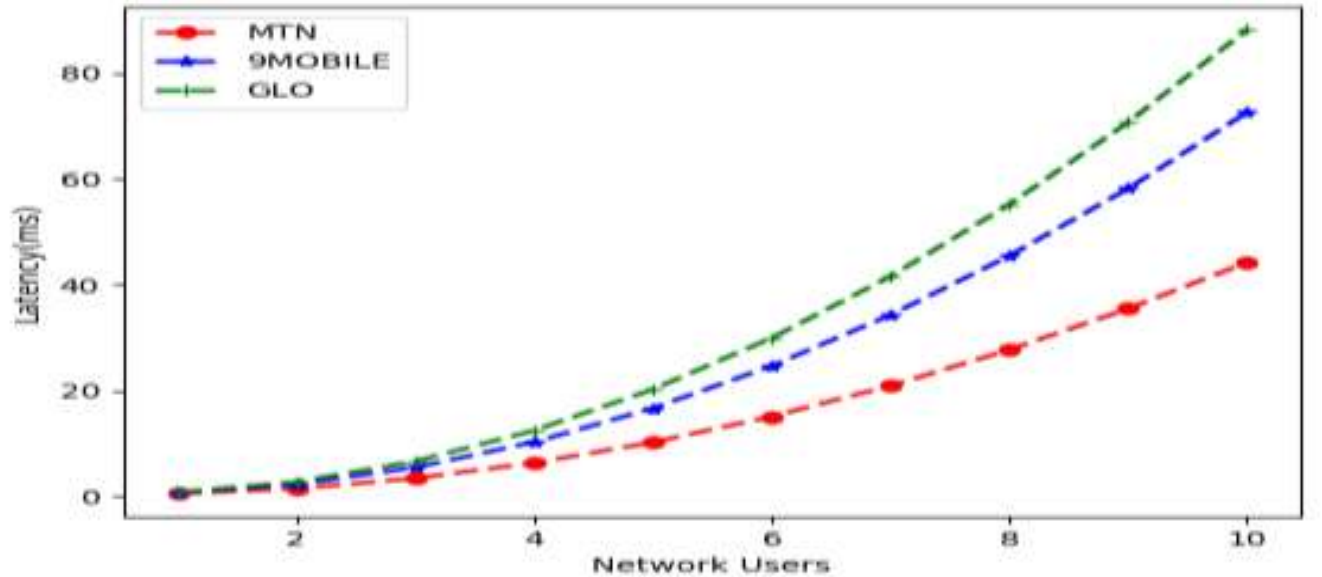

Figure 5: Latency of $4 \mathrm{G}$ Wi-Fi Network

Observation from figure 5 showed that latency increases as the number of network users increases. It also showed that MTN network has the lowest latency compared to GLO and 9MOBILE. The packet loss of the three mobile networks shown in table 2 was plotted and presented in figure 6

Table 2: Average Monthly Packet Loss in percentage for data transfer of 29.12MB using three Mobile Networks

\begin{tabular}{llll}
\hline & 4G NETWORK & & \\
\hline Users & MTN & 9MOBILE & GLO \\
\hline User1 & 0 & 0 & 0 \\
User2 & 0 & 0 & 0 \\
User3 & 0 & 0 & 0 \\
User4 & 0 & 0 & 0 \\
User5 & 0 & 0 & 0 \\
User6 & 0 & 0 & 0 \\
User7 & 0 & 0 & 0 \\
User8 & 0 & 0 & 0.8 \\
User9 & 0 & 0.87 & 1.07 \\
User10 & 1 & 1.2 & 1.53 \\
\hline
\end{tabular}

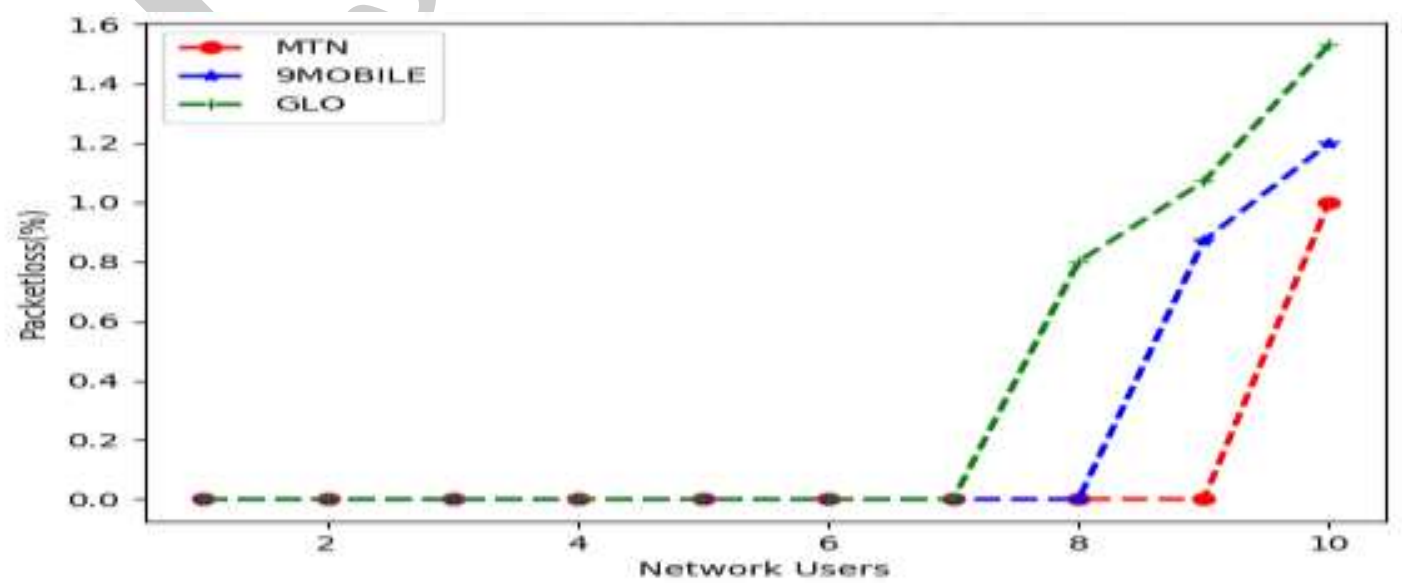

Figure 6: Packet loss of Existing Wi-Fi Network 
Figure 6 showed that there was packet loss from the seventh user for the GLO network, eighth user for the 9MOBILE network and ninth user for the MTN network. Table 3 and Figure 7 showed the results of the data throughput calculated from the developed program in Python 3.6.1 64Bit

Table 3: Average monthly Throughput in Mbps for data transfer of 29.12MB using three Mobile Networks

\begin{tabular}{llll}
\hline \multicolumn{4}{c}{ 4G NETWORK } \\
\hline Users & MTN & 9MOBILE & GLO \\
\hline User1 & $4.36581709 \mathrm{e}+08$ & $3.34648695 \mathrm{e}+08$ & $2.90763854 \mathrm{e}+08$ \\
User2 & $1.54989764 \mathrm{e}+08$ & $1.01658230 \mathrm{e}+08$ & $8.49846782 \mathrm{e}+07$ \\
User3 & $6.76435924 \mathrm{e}+07$ & $4.25000912 \mathrm{e}+07$ & $3.51846613 \mathrm{e}+07$ \\
User4 & $3.92863166 \mathrm{e}+07$ & $2.26888465 \mathrm{e}+07$ & $1.87253785 \mathrm{e}+07$ \\
User5 & $2.27642276 \mathrm{e}+07$ & $1.39935498 \mathrm{e}+07$ & $1.15318341 \mathrm{e}+07$ \\
User6 & $1.54450701 \mathrm{e}+07$ & $9.46020084 \mathrm{e}+06$ & $7.79125223 \mathrm{e}+06$ \\
User7 & $1.11449184 \mathrm{e}+07$ & $6.81212017 \mathrm{e}+06$ & $5.60789014 \mathrm{e}+06$ \\
User8 & $8.41233438 \mathrm{e}+06$ & $5.13028312 \mathrm{e}+06$ & $4.22627708 \mathrm{e}+06$ \\
User9 & $6.57089193 \mathrm{e}+06$ & $4.00772988 \mathrm{e}+06$ & $3.29782350 \mathrm{e}+06$ \\
User10 & $5.27264839 \mathrm{e}+06$ & $3.21383228 \mathrm{e}+06$ & $2.64421385 \mathrm{e}+06$ \\
\hline
\end{tabular}

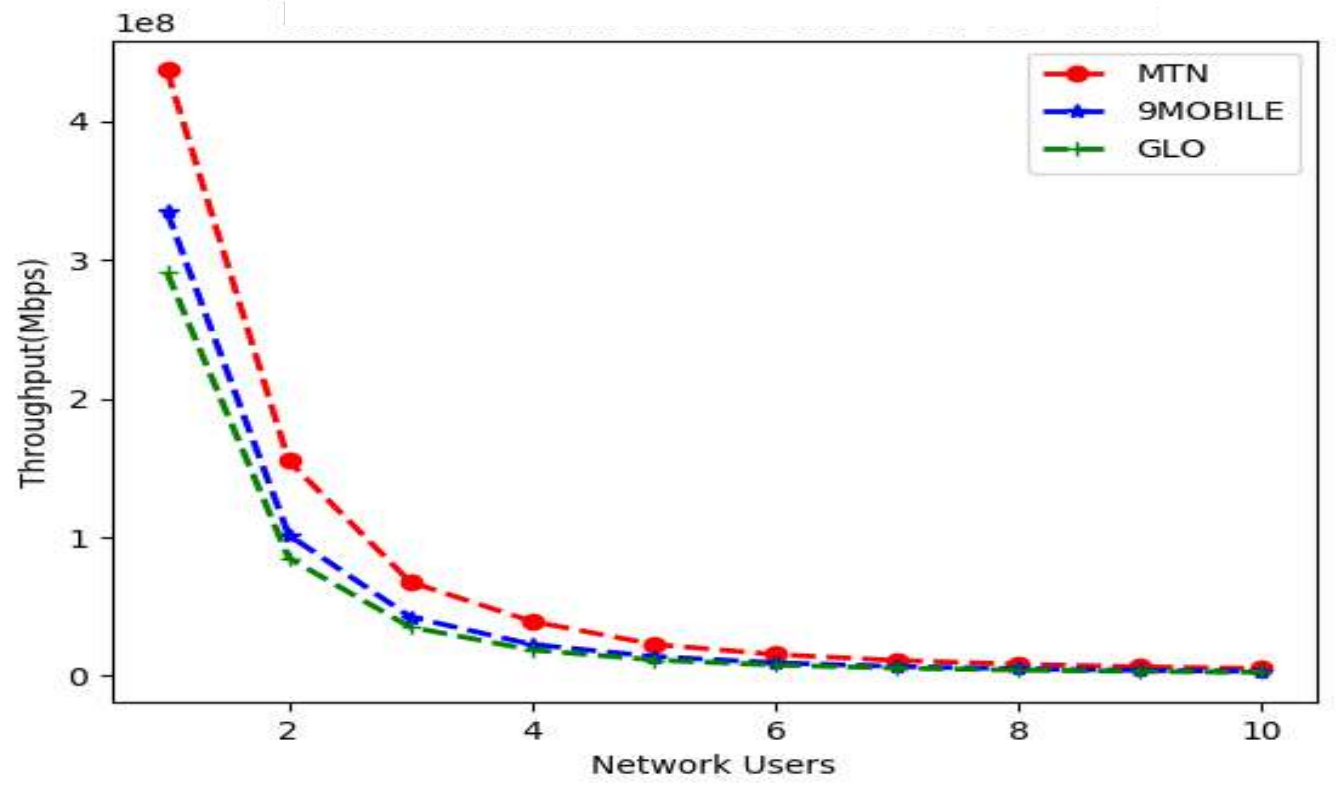

Figure 7: 4G Wi-Fi Throughput

Observation from figure 7 showed that when users connected to the various network steps up, the data throughput reduces, and this frustrates the users from downloading data from the network. It also showed that out of the three mobile networks, MTN got the best throughput, latency, and packet loss when users connected to the $3 \mathrm{G}$ Wi-Fi Network increases.

\section{Conclusion}

Wireless Fidelity is a technology that uses radio waves to provide internet connections. The evaluation of wireless fidelity performance showed that many users connected to (Wi-Fi) hotspot experiences the slow speed of the network, which leads to long processing hours, a decrease of network throughput and increases in latency. Since the WiFi network in Nigeria 
cannot provide fast and reliable data transmission due to congestion, other frequencies can be used to complement the existing radio frequencies in accessing the internet. Visible light spectrum can be used for communication at very high speed since today's fast-paced cloud computing era needs an excellent network performance.

\section{ACKNOWLEDGMENTS}

The authors wish to thank Enugu State University of Science and Technology for providing the Laboratory and equipment for the accomplishment of this research.

\section{REFERENCES}

[1] Nigeria Communications Commission, 2018. Spectrum Frequency Management https://www.ncc.gov.ng/technology/spectrum/frequency-management [accessed May 24, 2018].

[2] Dinesh, B., Kiran, J., Manikanta, K., 2015. High throughput Wi-Fi Back Scatter. ACM Digital Library, 283-296.

[3] Jie, Z., HwaJong, K., GooYeon, L., Yong, L., 2013. Throughput Improvement of a Range-aware Wi-Fi network by Minimizing Signal Interference. IARIA Conference, The Sixth International Conference on Advances in Mesh Networks, ISBN:978-1-61208-299-8, pp. 20-25.

[4] Osama, M. A., Esam, A., Omar, M.H., 2017. Adaptive device to device communication using Wi-Fi Direct in Smart Cities. Journal Wireless Networks 23(7), 2197-2213.

[5] Kamil, M.S., 2009. The Performance Evaluation of OFDM Based WLAN. Diva Portal, pp.1-48. www.diva-portal.org/smah/get/diva2:830372/FULLTEXT01.pdf [accessed June 5, 2018].

[6] Bradley, M., 2017. Introduction to Wi-Fi Wireless Networking. LifeWire, https://www.lifewire.com/introduction-to-wi-fi-wireless-networking-818265 [accessed April $24,2018]$.

[7] Guo, Y., Zhang, S., Xiao, D., 2012. Overview of Wi-Fi Technology. The 2nd International Conference on Computer Application \& System Modelly, pp. 1293-1296.

[8] Renaud Larue-Langlois, R., 2017. What is Throughput?6 Best Tools to Measure Throughput, https://www.google.com/amp/s/www.addictivetips.com/netadmin/throughput/amp/[accessed March 8, 2018].

[9] ConceptDraw, 2015. Wireless router network diagram, https://www.conceptdraw.com/examples/wireless-router-network-diagram [accessed April $28,2018]$. 